\title{
SECADO INDUSTRIAL DEL PINO RADIATA. TIEMPO DE ESTERILIZACIÓN CON CALOR Y EVOLUCION TEMPORAL DE LA TEMPERATURA
}

\author{
INDUSTRIAL DRYING OF RADIATA PINE. HEAT STERILIZATION TIME AND WOOD \\ TEMPERATURE EVOLUTION TIME
}

\author{
Rubén A. Ananias ${ }^{1}$, Rodrigo Venegas ${ }^{2}$
}

\begin{abstract}
RESUMEN
En este trabajo se estudia el tiempo mínimo para esterilizar la madera mediante un tratamiento con calor a $56 / 30{ }^{\circ} \mathrm{C} / \mathrm{min}$ (tratamiento HT) y se analiza la evolución temporal de la temperatura interna durante el secado a la escala industrial de pino radiata bajo programas de secado convencional, acelerado y alta temperatura.

El diseño experimental incluye como variables la temperatura de los tratamientos iniciales en ambiente saturado y el espesor de la madera. Se analiza para los espesores desde 16 hasta $100 \mathrm{~mm}$ el tiempo requerido para satisfacer las exigencias de HT especificadas por la norma NIMF 15 y se interrelaciona la evolución temporal de la temperatura interna de la madera con diversos programas de secado.

El tiempo mínimo estimado para conseguir esterilizar la madera mediante el tratamiento con calor HT $56 / 30^{\circ} \mathrm{C} / \mathrm{min}$ varia entre 58 y 224 minutos en madera de 16 y $100 \mathrm{~mm}$ respectivamente. Al debutar el secado la temperatura interna evoluciona rápidamente hasta la temperatura de bulbo húmedo lo que favorece la aplicación de programas de secado convencional acelerado y de alta temperatura para cumplir con las exigencias de HT de la norma NIMF 15.
\end{abstract}

Palabras clave: Pino radiata, métodos de secado, tratamiento térmico, esterilización con calor, Chile.

\begin{abstract}
In this work were studied the heat sterilization time (HT) and the evolution time of the internal wood temperature during industrial drying of radiata pine.

The experimental design included as variable the air temperature of the initial treatments in saturated atmosphere and the wood thickness. The minimal time required to satisfy the demands of HT specify by the NIMF 15 standard between 16 and $100 \mathrm{~mm}$ is predicted. The interrelationship between internal wood temperature and various schedules drying are analyzed.

The minimal time to heat sterilization wood the HT condition during the industrial drying of the radiata pine was between 58 and 224 minutes for 16 and $100 \mathrm{~mm}$ of wood thickness respectively. The internal

\footnotetext{
Universidad del Bío-Bío, Fac. Ingeniería. Depto. Ing. en Maderas. Avda. Collao 1202, Concepción, Chile. ananias@ubiobio.cl

Autor para correspondencia: ananias@ubiobio.cl

Recibido: 08.08.2005. Aceptado: 11.10.2005.
}

${ }^{2}$ Universidad del Bío-Bío, Fac. Ingeniería. Depto. Ing. en Maderas. Avda. Collao 1202, Concepción, Chile. rvenegas@alumnos.ubiobio.cl
\end{abstract}


temperature tends quickly until the wet temperature in early stages of drying, it favorise the application of accelerated and high temperature schedules to satisfy the HT demand of the NIMF 15 standard.

Keywords: Radiata pine, drying methods, heat treatments, heat sterilization, Chile.

\section{INTRODUCCION}

El secado artificial es un tratamiento térmico que permite proteger y esterilizar la madera de pino radiata dependiendo de los programas de secado utilizados (combinación de temperatura de bulbo seco y temperatura de bulbo húmedo). En particular para el secado industrial de pino radiata se utilizan los programas de secado convencional (SC), comúnmente $70 / 50^{\circ} \mathrm{C} /{ }^{\circ} \mathrm{C}$, convencional acelerado (SCA) típicamente $90 / 60^{\circ} \mathrm{C} /{ }^{\circ} \mathrm{C}$ y secado por alta temperatura (SAT) tal como $120 / 70^{\circ} \mathrm{C} /{ }^{\circ} \mathrm{C}$.

Por otra parte, diversos autores han mostrado que el tratamiento con calor permite esterilizar la madera. Los estudios muestran bajo que condiciones (combinaciones de temperatura, espesor o diámetro, tiempo y tipo de ambiente) pueden ser eliminados (exterminados) de la madera hongos, insectos y nemátodos (Newbill and Morrell, 1991; Dwinel et al., 1994; Morrell, 1995; Simpson 2002a, 2002b; Simpson et al. 2002).

La combinación de una temperatura en el centro de la madera de $56^{\circ} \mathrm{C}$ durante 30 minutos de exposición $\left(56 / 30^{\circ} \mathrm{C} / \mathrm{min}\right)$ es una condición HT (tratamiento con calor) que permite esterilizar la madera de hongos, insectos y nematodos (Morrel, 1995). Tal condición HT es actualmente exigida por las normas internacionales para la exportación de madera para embalajes (Norma NIMF 15).

Algunos métodos térmicos y su nivel de efectividad para eliminar (exterminar) hongos, insectos y nematodos son presentados en la literatura (Morrel, 1995). Las principales variables que condicionan los tratamientos térmicos son la temperatura y el espesor de la madera. Más alta temperatura y menor espesor de la madera requiere menos tiempo de exposición y mayor espesor de la madera implica un aumento de la temperatura y del tiempo de secado para conseguir la condición HT (Newbil and Morrell 1991; Morrell, 1995), igualmente un ambiente mas saturado reduce el tiempo y la temperatura de secado para la obtención de tal condición HT (Simpson 2002a, 2002b; Simpson et al. 2003).

Por otra parte diversos modelos estadísticos (polinomios) o modelos de transporte de calor (ecuación de transporte de calor uni y bidimensional) han sido utilizados para predecir el comportamiento de la temperatura de la madera durante el tratamiento con calor. En particular los modelos estadísticospermiten estimar satisfactoriamente el tiempo de tratamiento térmico (Simpson et al., 2001Simpson 2001, 2002a, 2002b). Igualmente, la solución analítica de McLean y soluciones numéricas han sido usados para simular la evolución temporal de la temperatura interna de la madera y de esta manera predecir el tiempo que requiere una determinada madera para conseguir tal condición $\mathrm{HT}$ al interior de la madera (Bai and Garrahan 1984; Sahle-Demessie et al., 1994; Simpson, 2003; 2004).

En Chile para el caso del pino radiata, los programas de secado usuales en la industria son el secado a temperatura convencional SC $70 / 50$ o $70 / 40{ }^{\circ} \mathrm{C} /{ }^{\circ} \mathrm{C}$, el secado convencional acelerado SCA 70/60, 80/ $60,90 / 60$ o $100 / 70{ }^{\circ} \mathrm{C} /{ }^{\circ} \mathrm{C}$ y el secado por alta temperatura SAT $110 / 70,120 / 70,125 / 75$ o $140 / 90{ }^{\circ} \mathrm{C} /{ }^{\circ} \mathrm{C}$. En todos estos casos el programa de secado se inicia con una calentamiento inicial con la mínima diferencia sicrométrica hasta la temperatura de bulbo húmedo y posteriormente un aumento de la temperatura ambiente hasta la temperatura del programa respectivo. 
Este trabajo tiene como objetivo estimar el tiempo mínimo de secado para cumplir con las exigencias de tratamiento térmico HT especificados por la norma NIMF 15 y analizar la evolución temporal de la temperatura interna de la madera durante el secado industrial de pino radiata.

\section{METODOLOGIA}

Este trabajo es realizado a la escala industrial en hornos de secado de $100 \mathrm{~m}^{3}$ de capacidad. El pino radiata es recogido en verde, recién aserrado y preparado para el secado en seis plantas industriales de la Octava Región de Chile (Figura 1).

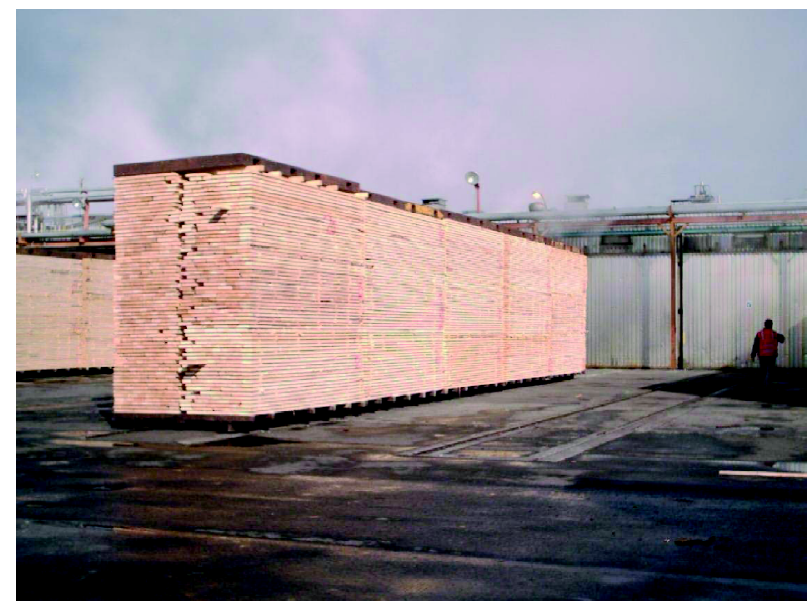

Figura 1: Planta de secado industrial con hornos de $100 \mathrm{~m} 3$ de capacidad.

La temperatura interna de la madera fue medida mediante 4 termocuplas insertadas en el centro de la madera (Figura 2a), las mediciones de la temperatura son recogidas a través de un sistema de adquisición de datos (Figura 2b) y almacenadas en un computador personal. Las termocuplas son debidamente calibradas antes de ejecutar cada ensayo, mediante un calibrador electrónico de termocuplas.

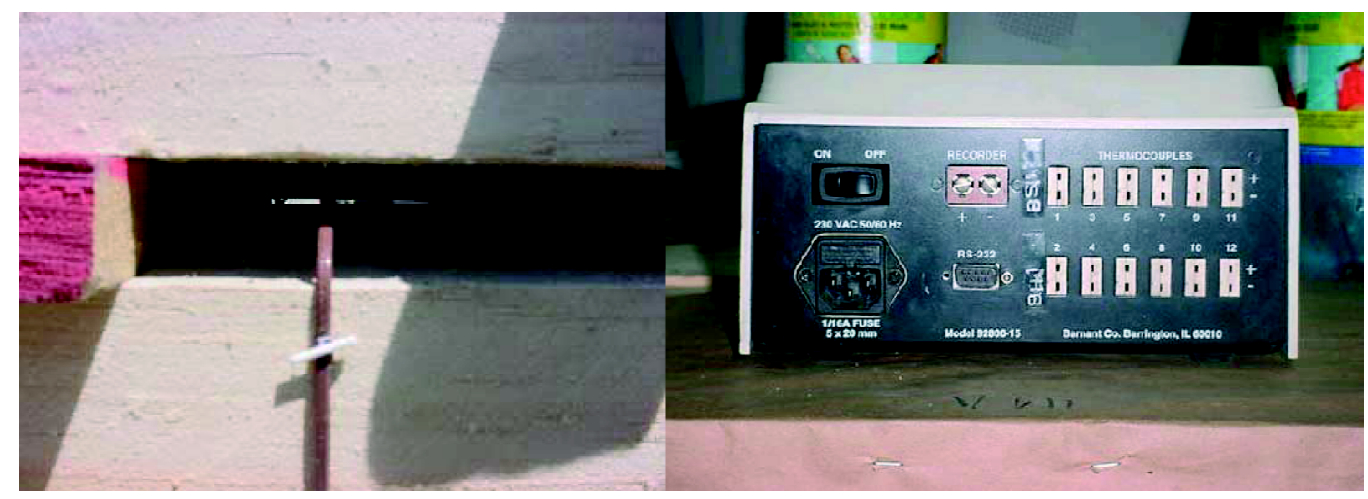

a)

b)

Figura 2: Instalación de termocuplas entre las camadas de madera y sistema de adquisición de datos de la temperatura. 
El tiempo de la adquisición de los datos vario entre 1 y 10 minutos dependiendo de las condiciones de secado y del espesor de la madera. Para la madera de $16 \mathrm{~mm}$ tratada a $100 / 100^{\circ} \mathrm{C} /{ }^{\circ} \mathrm{C}$ la variación del tiempo es de 1 minuto; para la madera de $92 \mathrm{~mm}$ tratada a $70 / 70^{\circ} \mathrm{C} /{ }^{\circ} \mathrm{C}$, el tiempo de adquisición de datos es de 10 minutos. Se desea obtener el tiempo mínimo cumplido $56 / 30\left({ }^{\circ} \mathrm{C} / \mathrm{min}\right)$, o sea cuando la temperatura interna de la madera es de $56^{\circ} \mathrm{C}$ y mantenida por lo menos 30 minutos.

\section{Ensayos para la predicción del tiempo mínimo de tratamiento HT}

Para la predicción del tiempo de tratamiento HT 56/30 $\left({ }^{\circ} \mathrm{C} / \mathrm{min}\right)$, el diseño experimental incluye los siguientes factores variables: a) temperatura calentamiento inicial $\left(70 / 70,80 / 80,90 / 90\right.$ y $100 / 100{ }^{\circ} \mathrm{C} /$ ${ }^{\circ} \mathrm{C}$ ) y el espesor de la madera : 16, 24 y $37 \mathrm{~mm}$. Son realizadas además, tres repeticiones en cada caso. En total son ejecutados 36 ensayos de secado de pino radiata a la escala industrial. El espesor de los separadores y la velocidad del aire no son consideradas variables y son constantes en este trabajo. Las predicciones son realizadas mediante algoritmos de regresión, utilizando el software Statgraphics plus 3.1.

\section{Evolución de la temperatura interna de la madera}

En los diversos ensayos de secado se analizó la evolución temporal de la temperatura interna de la madera. Los programas de secado utilizados fueron: secado convencional $\mathrm{SC} 70 / 50$ y $70 / 45^{\circ} \mathrm{C} /{ }^{\circ} \mathrm{C}$, secado convencional acelerado SCA $70 / 60,80 / 60,90 / 60,100 / 70,100 / 65^{\circ} \mathrm{C} /{ }^{\circ} \mathrm{C}$ y secado por alta temperatura SAT $120 / 70,125 / 75^{\circ} \mathrm{C} /{ }^{\circ} \mathrm{C}$. Los espesores considerados varían entre 16 y $92 \mathrm{~mm}(16,24$, $37,50$ y $92 \mathrm{~mm})$.

\section{RESULTADOS Y DISCUSION}

\section{Predicción del tiempo mínimo para esterilizar la madera con calor (tratamiento HT)}

Un modelo polinomial en función de la temperatura y el espesor de la madera es propuesto para representar el tiempo mínimo de esterilización de la madera con calor a $56 / 30^{\circ} \mathrm{C} / \mathrm{min}$ (tratamiento HT). La ecuación de regresión múltiple que predice el tiempo mínimo cumplido para esterilizar la madera mediante el tratamiento HT, tiene la forma siguiente:

$$
t_{H T}=109.3-0.78 \cdot T+1.7 \cdot e
$$

Siendo $T$ la temperatura del ambiente en ${ }^{\circ} \mathrm{C}$ y $e$ el espesor de la madera en $\mathrm{mm}$. La ecuación 1 tiene un $\mathrm{r}^{2}$ de 0.81 , con un nivel de confianza del $99 \%(\mathrm{p}<0.01)$.

La Figura 3 muestra las estimaciones del tiempo mínimo requerido para que la madera cumpla con la combinación de $56 / 30^{\circ} \mathrm{C} / \mathrm{min}$ en el centro de la madera, según la temperatura de calentamiento inicial utilizada en cada programa de secado (condición HT). Se aprecia que el tiempo disminuyó con la intensidad del calentamiento inicial. El tiempo estimado vario desde 58 minutos para la madera de $16 \mathrm{~mm}$ de espesor tratada bajo un calentamiento $100 / 100{ }^{\circ} \mathrm{C} /{ }^{\circ} \mathrm{C}$ a 224 minutos para la madera de 100 $\mathrm{mm}$ tratada bajo condiciones $70 / 70{ }^{\circ} \mathrm{C} /{ }^{\circ} \mathrm{C}$. Tales variaciones son consecuencia de la velocidad a la que la temperatura calienta desde la superficie al interior de la madera.

El incremento de la temperatura en el interior de la madera esta relacionada con la temperatura del bulbo húmedo utilizada en los tratamientos de calentamiento inicial. Esto es, el uso de una $\mathrm{T}_{\mathrm{H}}$ igual o superior a $70^{\circ} \mathrm{C}$ favorece el calentamiento rápido de la madera a esta temperatura (Figura 4). El aumento de la temperatura reduce la resistencia externa al calentamiento de la madera por una parte y por otra mejora la conducción del calor a través de la estructura interna de la madera, lo cual es a su vez 
favorecido por la alta humedad de la madera que facilita el transporte de calor por conducción al interior de la madera.

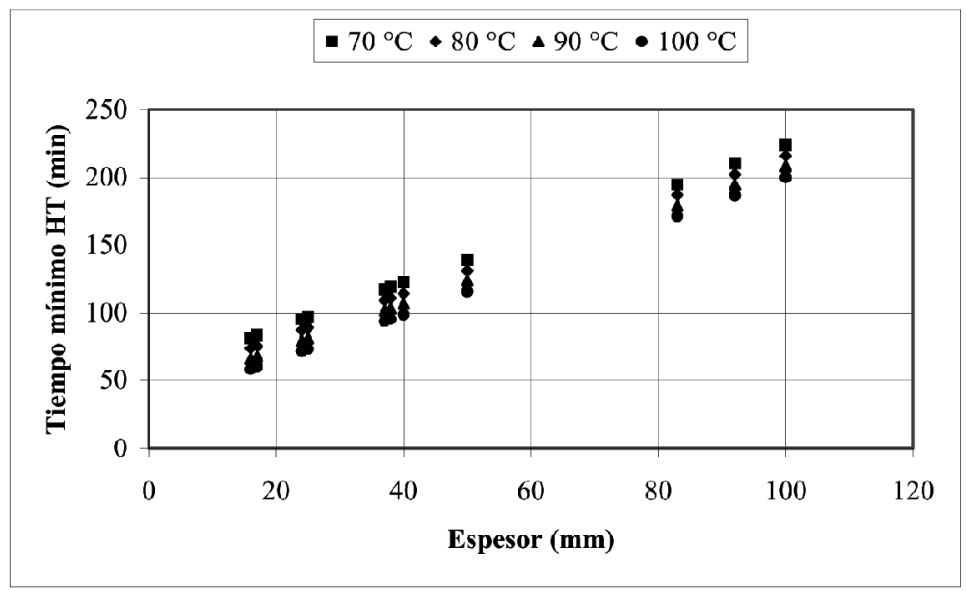

Figura 3: Tiempo mínimo (min) para obtener la condición HT $\left(56 / 30^{\circ} \mathrm{C} / \mathrm{min}\right)$ segœn temperatura ambiente y espesor de la madera.

La tabla 1 muestra los resultados experimentales y estimados del tiempo mínimo de tratamiento HT. Se observa que los datos estimados por la ecuación de regresión múltiple mostrada en la ecuación 1 se ajustan satisfactoriamente a los resultados experimentales, la desviación estándar es de $8.1 \%$, esta misma tendencia es dable esperar para mayores espesores. En la Tabla 1 se presenta además, el efecto del espesor y la temperatura de calentamiento sobre el tiempo mínimo de tratamiento HT. El espesor influye significativamente en el tiempo de tratamiento térmico HT y la temperatura afecta significativamente al tiempo de mínimo de tratamiento HT entre 70 y $90^{\circ} \mathrm{C}$, en cambio el tiempo mínimo de tratamiento térmico $\mathrm{HT}$ entre 90 y $100^{\circ} \mathrm{C}$ no es significativo. Esto último resulta interesante en las prácticas industriales de calentamiento inicial, ya que un ambiente saturado a $90^{\circ} \mathrm{C}$ consume menos energía térmica que el vapor saturado a $100^{\circ} \mathrm{C}$.

Tabla 1: Tiempo mínimo para esterilizar la madera mediante tratamiento con calor HT (min)

\begin{tabular}{|c|c|c|c|c|c|c|c|c|c|}
\hline \multirow[t]{2}{*}{ Espesor } & \multirow{2}{*}{$\begin{array}{l}\text { Significancia } \\
\text { estadística }\end{array}$} & \multicolumn{4}{|c|}{$\begin{array}{c}\text { Experimental } \\
\mathrm{T}^{\circ} \mathrm{C}\end{array}$} & \multicolumn{4}{|c|}{$\begin{array}{c}\text { Estimado } \\
\mathbf{T}^{\circ} \mathrm{C}\end{array}$} \\
\hline & & 71 & 80 & 90 & 100 & 70 & 80 & 90 & 1011 \\
\hline \multirow{3}{*}{16} & \multirow{3}{*}{$a$} & 75 & 68 & 68 & 56 & \multirow{3}{*}{82} & \multirow{3}{*}{74} & \multirow{3}{*}{66} & \multirow{3}{*}{59} \\
\hline & & 77 & 65 & 60 & 64 & & & & \\
\hline & & 69 & 70 & 65 & 58 & & & & \\
\hline \multirow{3}{*}{24} & \multirow{3}{*}{ b } & 107 & 85 & 86 & 78 & \multirow{3}{*}{96} & \multirow{3}{*}{88} & \multirow{3}{*}{80} & \multirow{3}{*}{72} \\
\hline & & 108 & 97 & 88 & 78 & & & & \\
\hline & & 110 & 88 & 82 & 80 & & & & \\
\hline \multirow{3}{*}{37} & \multirow{3}{*}{ c } & 107 & 115 & 110 & 90 & \multirow{4}{*}{118} & \multirow{4}{*}{110} & \multirow{4}{*}{102} & \multirow{4}{*}{94} \\
\hline & & 107 & 117 & 90 & 94 & & & & \\
\hline & & 129 & 110 & 90 & 83 & & & & \\
\hline \multicolumn{2}{|c|}{ Significancia estadística $T \longrightarrow$} & $d$ & e & $f$ & f & & & & \\
\hline
\end{tabular}




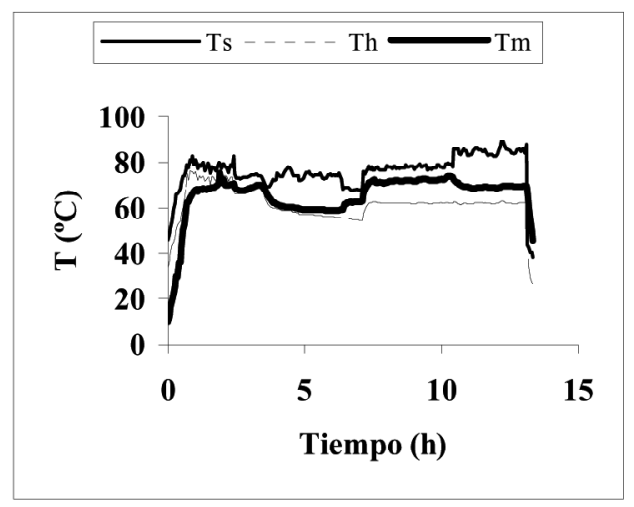

Ensayo $16 \mathrm{~mm}, 70 / 70{ }^{\circ} \mathrm{C} /{ }^{\circ} \mathrm{C}$

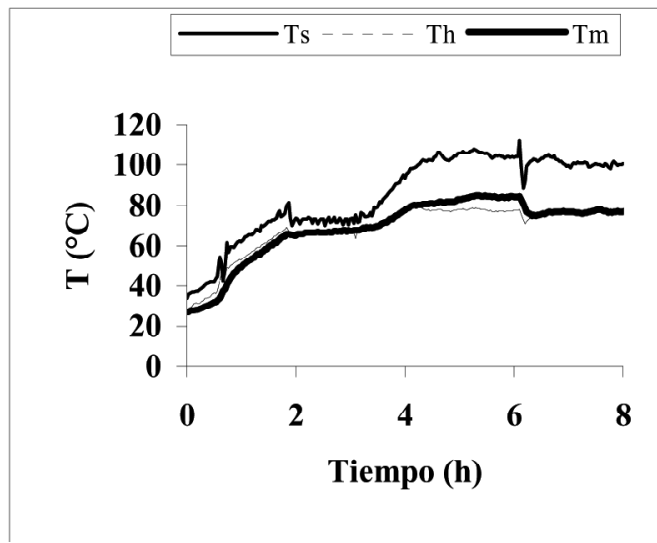

Ensayo $24 \mathrm{~mm}, 70 / 70{ }^{\circ} \mathrm{C} /{ }^{\circ} \mathrm{C}$

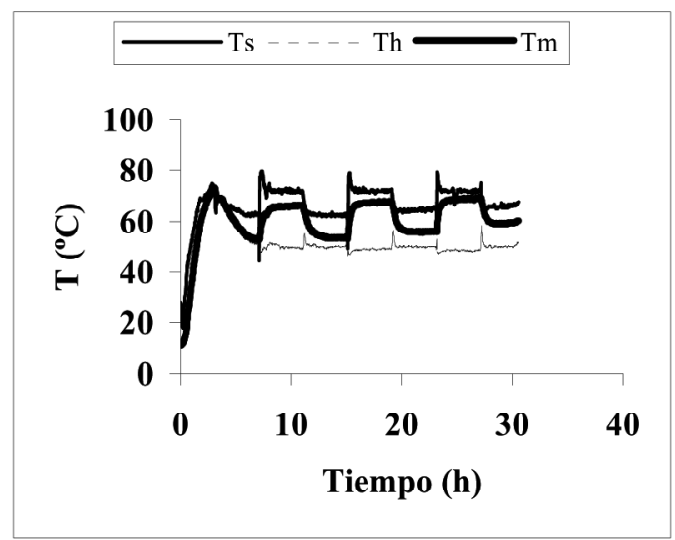

Ensayo $37 \mathrm{~mm}, 70 / 70{ }^{\circ} \mathrm{C} /{ }^{\circ} \mathrm{C}$

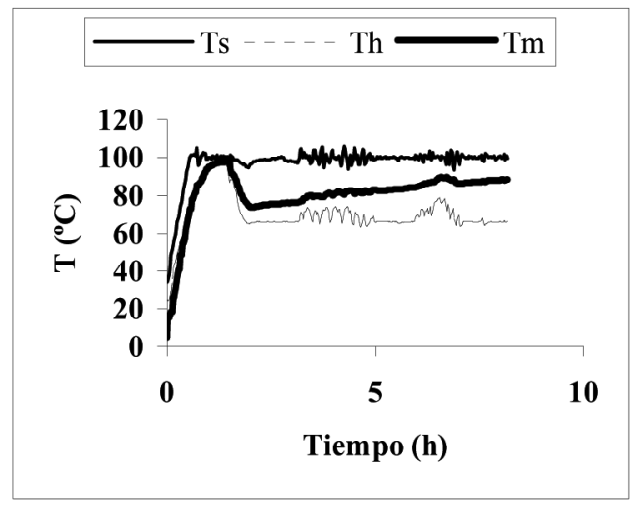

Ensayo $16 \mathrm{~mm}, 100{ }^{\circ} \mathrm{C} /{ }^{\circ} \mathrm{C}$

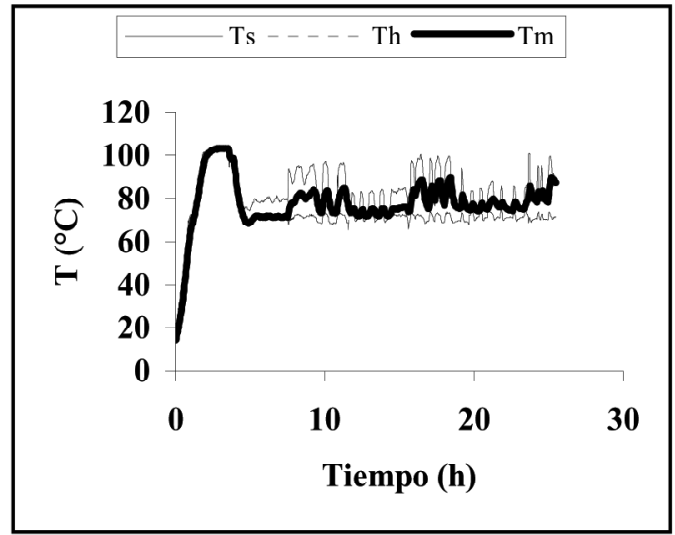

Ensayo $24 \mathrm{~mm}, 100 / 100{ }^{\circ} \mathrm{C} /{ }^{\circ} \mathrm{C}$

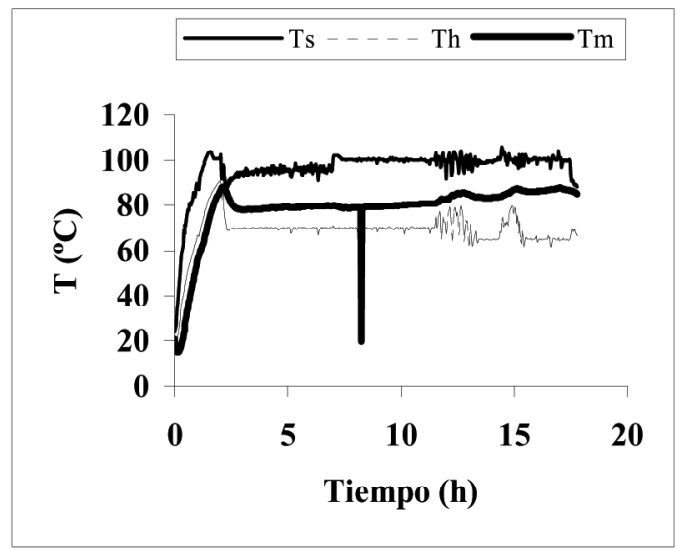

Ensayo $37 \mathrm{~mm}, 100 / 100{ }^{\circ} \mathrm{C} /{ }^{\circ} \mathrm{C}$

Figura 4: Programas de secado industrial con calentamiento inicial en ambiente saturado a diversas temperaturas.

( $\mathrm{Ts}=$ temperatura de bulbo seco. $\mathrm{Th}=$ temperatura de bulbo húmedo. Tm. Temperatura en el centro de la madera) 


\section{Evolución de la temperatura interna de la madera}

En la Figura 5 se muestran los programas de secado utilizados en estos ensayos. Los programas SC $75 / 40{ }^{\circ} \mathrm{C} /{ }^{\circ} \mathrm{C}$, SCA $90 / 60{ }^{\circ} \mathrm{C} /{ }^{\circ} \mathrm{C}$ y SAT $125 / 75{ }^{\circ} \mathrm{C} /{ }^{\circ} \mathrm{C}$. Se observa que la madera de pino radiata es sometida al proceso de secado usando temperaturas ambientales de entre 90 y $125^{\circ} \mathrm{C}$ por un tiempo de entre $15 \mathrm{~h}$, con un espesor de $92 \mathrm{~mm}$ y un programa SC $125 / 755\left({ }^{\circ} \mathrm{C} /{ }^{\circ} \mathrm{C}\right)$ y 40 horas, con un espesor de $37 \mathrm{~mm}$ y un programa $90 / 60\left({ }^{\circ} \mathrm{C} /{ }^{\circ} \mathrm{C}\right)$. Además, dependiendo del programa de secado y el espesor de la madera, la temperatura interna de la madera llega a alcanzar unas temperaturas máximas de entre $80 \mathrm{y}$ $100{ }^{\circ} \mathrm{C}$ por tiempo prolongado.

Se observa que el programa SC $75 / 40{ }^{\circ} \mathrm{C} /{ }^{\circ} \mathrm{C}$ para $50 \mathrm{~mm}$ el calentamiento inicial permite llevar la temperatura interna hasta cerca de $70^{\circ} \mathrm{C}$ luego esta condición es mantenida por el tiempo necesario para obtener la condición de HT exigida por la norma NIMF 15. Posteriormente la temperatura interna de la madera baja hasta alrededor de $50^{\circ} \mathrm{C}$ manteniéndose en esta condición durante el tiempo restante de secado.

Cuando el programa de secado es más intenso tal como en el $\mathrm{SCA} 90 / 60^{\circ} \mathrm{C} /{ }^{\circ} \mathrm{C}$ para $37 \mathrm{~mm}$, la temperatura interna de la madera sube rápidamente hasta la temperatura de bulbo húmedo, esto es alrededor de $\operatorname{los} 60^{\circ} \mathrm{C}$, temperatura que sigue aumentando en el transcurso del secado por unas 36 horas hasta alrededor de $90^{\circ} \mathrm{C}$.

En el proceso SAT $125 / 75^{\circ} \mathrm{C} /{ }^{\circ} \mathrm{C}$, para un espesor de la madera de $92 \mathrm{~mm}$ la temperatura de la madera demora en alcanzar la temperatura del bulbo húmedo, esto es $75^{\circ} \mathrm{C}$, pero puede llegar posteriormente hasta cerca de $\operatorname{los} 100^{\circ} \mathrm{C}$ hacia el final del secado.

El comportamiento de la temperatura interna de la madera se fundamenta teóricamente por una parte por la alta permeabilidad del pino radiata que favorece el movimiento interno de liquido hasta la interfase de la madera y el calor aportado por el programa es usado principalmente para evaporar el agua en la superficie, imponiéndose probablemente una primera etapa de secado constante hasta que deja de llegar suficiente liquido a la interfase y comienza entonces el calentamiento de la madera a una temperatura mayor que la $\mathrm{T}_{\mathrm{H}}$ (Ananías et al., 2005)

Teóricamente la superficie de la madera y toda la madera alcanzan durante la primera etapa de secado la temperatura de bulbo húmedo y toda la evaporación ocurre al nivel de la superficie de la madera, toda la energía se ocupa inicialmente para calentar la madera, el aire y los elementos del horno de secado. El calor adicional es utilizado enteramente para evaporar el agua en la superficie de la madera. En tal condición la madera se encuentra a la temperatura de bulbo húmedo. Por lo que en los programas SCA $80 / 60$ y $100 / 70$ y SAT $120 / 70{ }^{\circ} \mathrm{C} /{ }^{\circ} \mathrm{C}$, el centro de la madera alcanza muy rápidamente $\operatorname{los} 60^{\circ} \mathrm{C}$, favoreciéndose con ello el logro de las condiciones exigidas por la norma NIMF 15 . Este comportamiento puede extenderse por analogía a los programas $\mathrm{SCA} 70 / 60$ y $90 / 60^{\circ} \mathrm{C} /{ }^{\circ} \mathrm{C}$ y SAT $110 /$ $70,125 / 75$ y $140 / 90^{\circ} \mathrm{C} /{ }^{\circ} \mathrm{C}$.

En el proceso SAT $125 / 75^{\circ} \mathrm{C} /{ }^{\circ} \mathrm{C}$, para un espesor de la madera de $92 \mathrm{~mm}$ la temperatura de la madera demora en alcanzar la temperatura del bulbo húmedo, en este caso no llega suficiente liquido hasta la superficie de la madera y se impone rápidamente un gradiente de humedad en la superficie de la madera y el frente de evaporación se desplaza hacia el interior de la madera..

Lo anteriormente expuesto refuerza que los programas SCA y SAT de la madera aserrada de pino radiata cumplen ampliamente con las disposiciones indicadas por la norma NIMF 15, esto es cuando la madera aserrada de pino radiata es secada bajo programas SCA 70/60, 80/60, 90/60 y 100/70 o SAT $120 / 70$ y $125 / 75^{\circ} \mathrm{C} /{ }^{\circ} \mathrm{C}$, la temperatura interna de la madera alcanza rápidamente los $60^{\circ} \mathrm{C}$ o más y posteriormente durante el secado la madera según el programa SCA o SAT llega a temperaturas de 
entre $70 \mathrm{y}$ mas de $100^{\circ} \mathrm{C}$, lo que hace innecesario la aplicación de tratamientos térmicos con vapor o realizar mediciones in-situ de la temperatura de la madera. En cambio bajo un programa de $\mathrm{SC}$ se debe conseguir la condición HT en el calentamiento inicial.

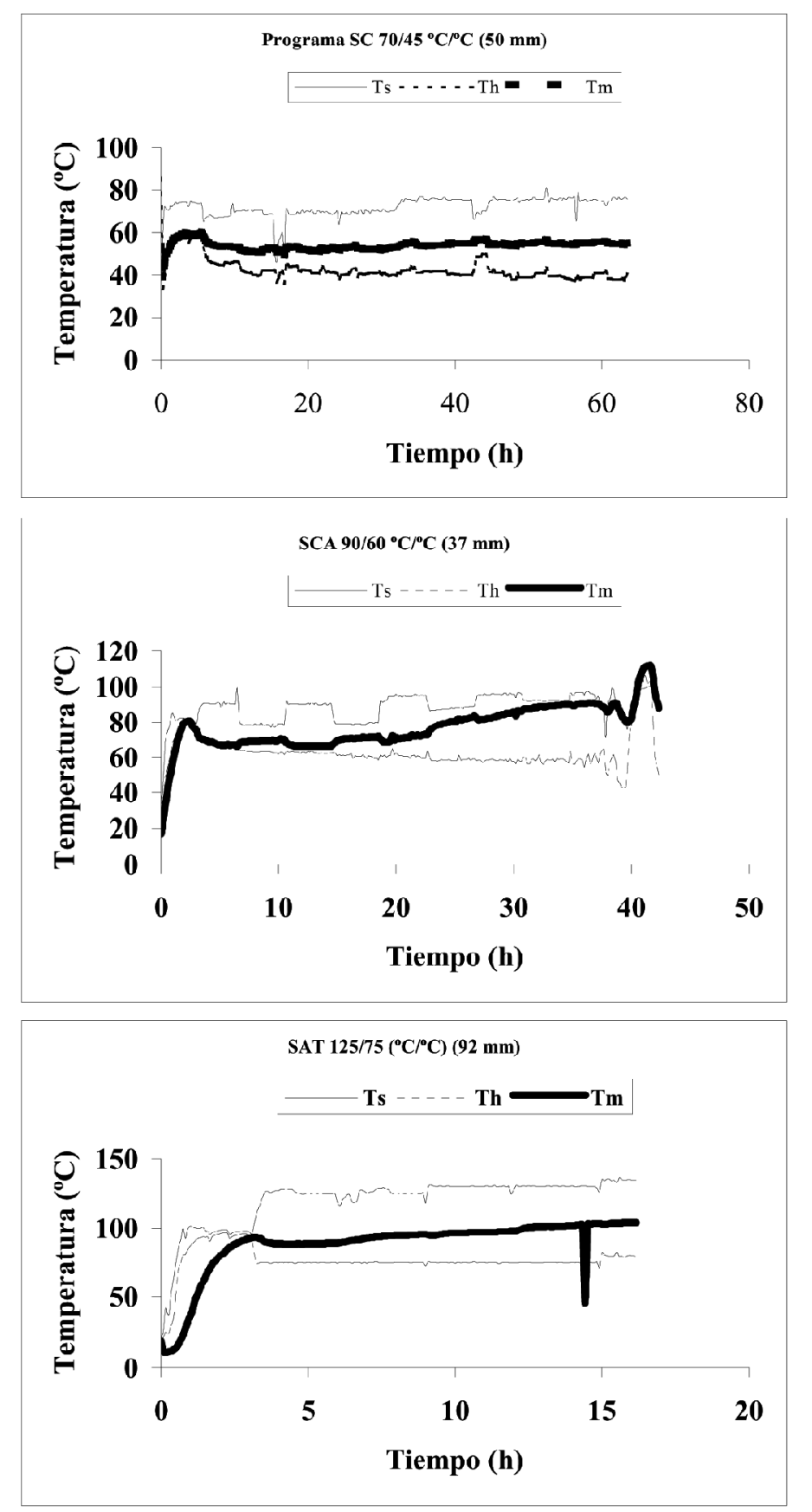

Figura 5: Programas de secado industrial de pino radiata

$\mathrm{Ts}=$ temperatura de bulbo seco. $\mathrm{Th}=$ temperatura de bulbo húmedo. $\mathrm{Tm}=$ temperatura en el centro de la madera 


\section{CONCLUSIONES}

El tiempo mínimo para obtener las condiciones HT exigidas por la norma NIMF es estimado entre 58 minutos en la madera de $16 \mathrm{~mm}$ de tratada $100 / 100^{\circ} \mathrm{C} /{ }^{\circ} \mathrm{C}$ hasta 224 minutos en madera de $100 \mathrm{~mm}$ de espesor tratada $70 / 70^{\circ} \mathrm{C} /{ }^{\circ} \mathrm{C}$.

De acuerdo a la evolución temporal de la temperatura interna de la madera, los programas de secado industrial del pino radiata SCA $80 / 60$ y $100 / 70^{\circ} \mathrm{C} /{ }^{\circ} \mathrm{C}$ y SAT $120 / 70^{\circ} \mathrm{C} /{ }^{\circ} \mathrm{C}$ son valorizados como medio de tratamiento térmico y cumplen satisfactoriamente con las especificaciones de HT de la norma NIMF 15. Esta valorización puede ser extendida por analogía a los programas SCA $70 / 60$ y $90 / 60^{\circ} \mathrm{C} /$ ${ }^{\circ} \mathrm{C}$ y SAT $110 / 70,125 / 75$ y $140 / 90^{\circ} \mathrm{C} /{ }^{\circ} \mathrm{C}$.

Los programas $\mathrm{SC} 70 / 45,70 / 50^{\circ} \mathrm{C} /{ }^{\circ} \mathrm{C}$ requieren mantener por un tiempo pertinente el calentamiento inicial a la temperatura máxima del secado para obtener la condición HT de la NIMF 15. Este comportamiento puede ser extendido por analogía a todos los programas de SC cuya temperatura de bulbo húmedo es inferior a $60^{\circ} \mathrm{C}$.

\section{AGRADECIMIENTOS}

A la Ing. Linette SALVO del Depto. de Ingeniería en Maderas de la Universidad del Bío-Bío, por su colaboración en el tratamiento estadístico de los datos experimentales.

\section{BIBLIOGRAFIA}

Ananías, R.A.; Vallejos, S.; Salinas, C. 2005. Estudio de la cinética del secado convencional y bajo vacío del pino radiata. Maderas: Ciencia y Tecnología 7(1):37-47.

Bai, G.L.; Garrahan, P. 1984. The temperature and moisture content in lumber during preheating and drying. Wood Science and Technology 18:121-135.

Dwinel, L.D.; Avramidis, S.; Clark, J.E. 1994. Evaluation of a radio-frequency/vacuum dryer for eradicating the pinewood nematode in green sawn wood. Forest Products Journal 44(4):19-24.

Morrel, J.J. 1995. Importation of unprocessed logs into north America: A review of pest mitigation procedures and their efficacy. Forest Products Journal 45(9):41-50.

Newbill, M.A.; Morrel, J.J. 1991. Effect of elevated temperatures on survival of Basidiomycetes that colonise untreated Douglas-fir poles. Forest Products Journal 41(6):31-33.

NIMF 15. 2002. Directrices para reglamentar el embalaje de madera utilizado en el comercio internacional. Normas internacionales para medidas fitosanitarias. FAO, Roma. 18 p.

Sahle-Demessie, E.; Levien, K.L.; Morrel, J.J.; Newbill. 1992. Modelling internal temperature changes of timber poles during ACA treatment. Wood Science and Technology 26:227-240.

Simpson, W.T. 2004. Two-dimensional heat flow analysis applied to heat sterilisation of ponderosa pine and douglas-fir square timbers. Wood and Fiber Science 36(3):459-464. 
Simpson, W.T. 2003. Mechanism responsible for the effect of wet bulb depression on heat sterilisation of slash pine lumber. Wood and Fiber Science 35(2):175-186

Simpson, W.T. 2002 ${ }^{\mathrm{a}}$. Effect of wet bulb depression on heat sterilisation time of slash pine lumber. USDA-FPL, Research paper FPL-RP-6004. 6p.

Simpson, W.T. 2002b. Lumber drying and heat sterilisation research at the US Forest Products Laboratory. 30th Hardwood Symposium proceeding 73-75.

Simpson, W.T.; Wang, X.; Verril, S. 2002. Heat sterilisation time of ponderosa pine and douglasfir boards and square timbers. USDA-FPL, Research paper FPL-RP-607.24p.

Simpson, W.T. 2001. Heating times for round and rectangular cross sections of wood in steam. USDA-FPL, General technical report FPL-GTR-130. 103p. 